\title{
Robust Optimization of Road Vehicle Suspension Considering the Variation of Tire Vertical Stiffness
}

\author{
Liunan Yang, Federico Ballo, Giorgio Previati, Massimiliano Gobbi \\ Department of Mechanical Engineering, Politecnico di Milano, Italy \\ E-mail: massimiliano.gobbi@polimi.it
}

Received: 15 February 2019; Accepted: 7 March 2019; Available online: 1 May 2019

\begin{abstract}
Tire vertical stiffness is influenced by many factors. The inflation pressure, tire dimension, and usage of run-flat tire are considered in this paper. Robust multi-objective optimization technique is used to optimize the suspension performance considering the variation of the tire vertical stiffness. Three objective functions, discomfort, road holding, and working space are used to evaluate the dynamic behavior of the suspension considering a two-degree-of-freedom quarter-car model excited by a random road profile. The Pareto-optimal solutions in terms of suspension spring stiffness and damping coefficient are obtained and compared with the one computed by means of a deterministic approach. Solutions obtained by means of the robust optimization method are proven to be less sensitive to the possible variations of the tire vertical stiffness without influencing significantly the expected performance.
\end{abstract}

Keywords: Robust optimization; Multi-objective optimization; Passive suspension; Tire vertical stiffness; Runflat tire.

\section{Introduction}

Multi-objective optimization approaches are widely used to improve the vehicle performance. In particular, referring to the design of suspension systems, the dynamic behavior of passive and active suspensions has been optimized in [1-4]. On the basis of simple analytical models, these studies provide a useful guidance on selecting the optimal suspension parameters to maximize the vehicle performances in terms of both comfort and handling. However, in real-world situations, mechanical components are affected by uncertainties and their behavior may not be exactly the same as the expected one. In the case of suspension systems, spring stiffness and damping coefficient may differ from their nominal values due to uncertainties related to the manufacturing process $[5,6]$. Moreover, it is well recognized in the literature, that solutions obtained from deterministic optimization are more sensitive to uncertainties on the suspension parameters [7]. To overcome this limitation, multi-objective robust optimization has been successfully employed in the design of suspension systems [8-10].

Tire behavior is outmost important for the vehicle active safety [11] and it plays a crucial role in defining suspensions behavior. Given its importance, it is easy to understand that any deviation from the nominal behavior strongly affects the performance of the suspension system (and of the entire vehicle). The vertical stiffness of the tire for instance, strongly depends on the inflation pressure and can assume significantly different values even within the range of allowable tire pressures. The structure of the tire itself is another important parameter that affects its vertical stiffness. If run-flat tires are considered, a higher vertical stiffness is shown if compared with conventional tires with the same size. In addition, the wide range of optional tire size provided by vehicles manufactures can also lead to different tire vertical stiffness.

As both comfort and handling are sensitive to variations of tire vertical stiffness [1], the present paper aims at analyzing the problem of discomfort, road holding, and working space minimization in a multi-objective robust optimization framework. The simple two degrees of freedom (DOF) quarter car model is employed to describe the vertical dynamics of the vehicle running on rough surfaces; such a model, despite its simplicity, provides a reasonable accuracy in modelling the vehicle dynamic behavior in the vertical direction [12]. Suspension spring and damping coefficients are considered as design parameters to be optimized; multi-objective robust optimization approach is applied by accounting for uncertainty sources affecting the tire vertical stiffness parameter.

The paper is organized as follows. Firstly, the mathematical quarter-car model is introduced and described in section 2. Then in section 3, the deterministic and robust multi-objective optimization approaches are described and the equations of the objective functions are derived. Finally, in section 4 the Pareto-optimal solutions obtained with the two optimization approaches are presented and discussed. 


\section{Vehicle model}

The simple quarter-car model shown in Figure 1 is employed for describing the vertical motion of a vehicle subject to a road irregularity excitation [1]. The model consists of the unsprung mass $\left(m_{1}\right)$, sprung mass $\left(m_{2}\right)$, tire vertical stiffness $\left(k_{1}\right)$, external road excitation $(r)$. Suspension stiffness and damping are defined by $k_{2}$ and $r_{2}$ respectively.

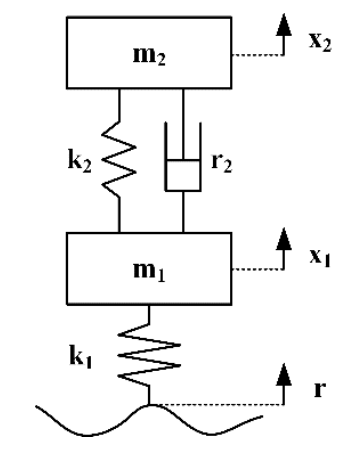

Figure 1. Quarter-car model.

The equations of motions of the quarter-car model are given in Equation (1),

$$
\begin{aligned}
& m_{1} \ddot{x}_{1}-r_{2}\left(\dot{x}_{2}-\dot{x}_{1}\right)-k_{2}\left(x_{2}-x_{1}\right)+k_{1}\left(x_{1}-r\right)=0 \\
& m_{2} \ddot{x}_{2}+r_{2}\left(\dot{x}_{2}-\dot{x}_{1}\right)+k_{2}\left(x_{2}-x_{1}\right)=0
\end{aligned}
$$

Road irregurality $(r)$ can be described by a random variable defined by a stationary and ergodic stochastic process with zero mean value [13]. The power spectral density (PSD) of the process can be derived either by experimental data, or by analytical formulae available in the literature. Among many formulations of the PSD available for example in [14], the single slope PSD defined by Equation (2) has been selected

$$
S_{r}(s)=\frac{A_{b} v}{\omega^{2}}
$$

where $A_{b}$ is a parameter that accounts for the road roughness, while $v$ represents the vehicle velocity. A typical compact sport car is considered as the reference vehicle and the relative parameters are summarized in Table 1 .

Table 1. Data of reference vehicle, running condition, and bounds for the design variables.

\begin{tabular}{cllll}
\hline Parameter & Unit & Reference value & Lower bound & Upper bound \\
\hline$m_{1}$ & $\mathrm{~kg}$ & 31 & - & - \\
$m_{2}$ & $\mathrm{~kg}$ & 229 & - & - \\
$k_{1}$ & $\mathrm{~N} / \mathrm{m}$ & 250230 & - & - \\
$k_{2}$ & $\mathrm{~N} / \mathrm{m}$ & 25000 & 0 & 80000 \\
$r_{2}$ & $\mathrm{Ns} / \mathrm{m}$ & 1000 & 0 & 5000 \\
$A_{b}$ & $\mathrm{~m}$ & $6.9 \mathrm{e}-6$ & - & - \\
$v$ & $\mathrm{~m} / \mathrm{s}$ & 20 & - & - \\
\hline
\end{tabular}

\section{Multi-objective optimization}

Multi-objective optimization approaches have been employed to find optimal values of suspension stiffness $k_{2}$ and damping $r_{2}$, denoted as design variables in the following. The objective functions to minimize are related to vehicle comfort and handling. In the following subsections a multi-objective optimization (MOO) problem is firstly formulated in the deterministic framework and then robustness of the system is considered by adding a source of uncertainty in the tire vertical stiffness $k_{1}$.

\subsection{Deterministic formulation}

A general MOO problem can be mathematically formulated as 


$$
\begin{aligned}
\min _{\mathbf{x} \in S} \mathbf{F}(\mathbf{x}) & =\mathbf{F}\left(F_{1}(\mathbf{x}), F_{2}(\mathbf{x}), \ldots, F_{k}(\mathbf{x})\right) \\
\text { s.t.G(} \mathbf{G}(\mathbf{x}) & =\left(g_{1}(\mathbf{x}), g_{2}(\mathbf{x}), \ldots, g_{w}(\mathbf{x})\right) \leq 0
\end{aligned}
$$

where $\mathbf{F}$ is the vector of objective functions, $\mathbf{X}$ is the vector of deign variables, and $\mathbf{G}$ is the vector of constraints.

Referring to the problem analyzed in this paper, the design variables to be optimized are the suspension equivalent stiffness $k_{2}$ and damping $r_{2}$; such design variables vary within a prescribed range, the relative lower and upper bounds are reported in Table 1 .

Objective functions are defined basing on the outputs of the quarter-car model. The outputs are the vehicle body vertical acceleration $\left(\ddot{x}_{2}\right)$, dynamic tire load $\left(F_{z}\right)$, and relative displacement between wheel and vehicle body $\left(x_{2}-\right.$ $\left.x_{1}\right)$. The objective functions to be minimized are the standard deviation of each output, namely, discomfort $\left(\sigma_{\ddot{x}_{2}}\right)$, road holding $\left(\sigma_{F_{z}}\right)$, and working space $\left(\sigma_{x_{2}-x_{1}}\right)$, respectively.

Based on the expressions of the three transfer functions $\left(H_{l}, l=1,2,3\right)$ between the road input and each output, which can be found in [1], the PSD of the output can be computed as in [15].

$$
S_{l}(\omega)=\left|H_{l}(j \omega)\right|^{2} S_{r}(\omega)
$$

where $H_{1}$ is the transfer function between $r$ and $\sigma_{\ddot{x}_{2}}, H_{2}$ is the transfer function between $r$ and $\sigma_{F_{z}}, H_{3}$ is the transfer function between $r$ and $\sigma_{x_{2}-x_{1}}$. The PSD $S_{l}(\omega), l=1,2,3$ corresponding to $H_{l}$.

By substituting Equation (2) into Equation (4) the following relation is obtained

$$
S_{l}(\omega)=A_{b} v\left|\omega^{-1} H_{l}(j \omega)\right|^{2}
$$

Thus, the variance of the random variable whose PSD is $S_{l}$ can be derived as

$$
\sigma_{l}^{2}=\frac{1}{2 \pi} \int_{-\infty}^{+\infty} S_{l}(\omega) d \omega
$$

where $\sigma_{1}, \sigma_{2}$, and $\sigma_{3}$ are the objective functions discomfort $\left(F_{1}\right)$, road holding $\left(F_{2}\right)$, and working space $\left(F_{3}\right)$ respectively. The analytical expressions of the objective functions are presented in Equations (8)(9)(10), and they are simplified by using the variables in Equation (7).

$$
q=\frac{m_{1}}{m_{2}}, \quad K=k_{2} \frac{(1+q)^{2}}{k_{1} q}, \quad R=r_{2} \sqrt{\frac{(1+q)^{3}}{k_{1} m_{2} q}}
$$

\section{Discomfort:}

$$
\begin{aligned}
& F_{1}=\sigma_{\ddot{x}_{2}}=\sqrt{\frac{1}{2} A_{b} v} f_{1} \\
& f_{1}=\frac{1}{R} \sqrt{\frac{m_{2}(1+q)^{5}}{k_{1} q}}
\end{aligned}
$$

\section{Road holding:}

$$
\begin{aligned}
& F_{2}=\sigma_{F_{z}}=\sqrt{\frac{1}{2} A_{b} v f_{2}} \\
& f_{2}=\left(\frac{K^{2}}{R}+\frac{R}{q}\right) \sqrt{\frac{k_{1}^{3} q^{3}}{m_{2}^{3}(1+q)^{3}}}
\end{aligned}
$$

\section{Working space:}

$$
\begin{aligned}
& F_{3}=\sigma_{x_{2}-x_{1}}=\sqrt{\frac{1}{2} A_{b} v} f_{3} \\
& f_{3}=\left(\frac{(K-1)^{2}}{R}+\frac{\left(1+R^{2}\right)}{q R}\right) \sqrt{k_{1}^{3} m_{2} q^{3}(1+q)}
\end{aligned}
$$




\subsection{Stochastic formulation}

Deterministic multi-objective optimization methods have been widely applied to solve mechanical engineering problems. However, since some actual parameters values may be different from their nominal values, the objective functions would have a stochastic nature. These can happen in the engineering problem, due to the manufacturing accuracy, operating conditions, etc.

The aim of a robust multi-objective optimization approach is to minimize the objective functions values along with their sensitivity to perturbations. In a design problem, parameters can be divided into two groups, namely design variables $(x)$ which are freely defined by the designers and noise factors $(c)$ not controlled by the designers.

Let us assume that the uncertainty of design variables $(x)$ and noise factors $(c)$ follow a normal distribution which can be described by mean values $\left(\mu_{x}, \mu_{c}\right.$ respectively) and standard deviations $\left(\sigma_{x}, \sigma_{c}\right.$ respectively). If all the input parameters are also uncorrelated, mean and standard deviation of each objective functions read [16]

$$
\begin{aligned}
& \mu_{F_{i}}=F_{i}\left(\mu_{z}, \mu_{c}\right) \\
& \sigma_{F_{i}}^{2} \approx \sum_{j=1}^{n}\left(\frac{\partial F_{i}}{\partial x_{j}}\right)^{2} \sigma_{x_{j}}^{2}+\sum_{j=1}^{m}\left(\frac{\partial F_{i}}{\partial c_{j}}\right)^{2} \sigma_{c_{j}}^{2}
\end{aligned}
$$

where $i=1,2,3$ are the deterministic objective functions, $m$ and $n$ are the number of design variables and noise factors respectively.

By assuming that the objective functions follow the normal distribution, a stochastic problem can be converted into an equivalent deterministic problem as follows. The designer can choose a level of risk $\beta_{i}$, representing the probability that the objective function is less than the computed value. Then, the stochastic objective functions can be written as a

$$
\begin{aligned}
& \bar{F}_{i}=\mu_{F_{i}}+\alpha_{i} \sigma_{F_{i}} \\
& \alpha_{i}=\Phi^{-1}\left(\beta_{i}\right)
\end{aligned}
$$

where the function $\Phi^{-1}$ is the inverse of the standard normal distribution. $\alpha_{i}$ are called robustness index of each objective function.

In this paper, only the variation of the tire vertical stiffness is considered. Therefore, the variance of objective function calculated by Equation (12) reads

$$
\sigma_{F_{i}}^{2}=\left(\frac{\partial F_{i}}{\partial k_{1}}\right)^{2} \sigma_{k_{1}}^{2}
$$

The mean value of the objective function $F_{i}$ can be computed by deterministic formulations considering the reference vehicle. Thus, the stochastic objective function $\bar{F}_{i}$ can be derived by applying Equation (13), and it reads

$$
\bar{F}_{i}=F_{i}+\alpha_{i} \sigma_{F_{i}}
$$

where $\bar{F}_{i}, i=1,2,3$ are the stochastic discomfort $\left(\bar{\sigma}_{\ddot{x}_{2}}\right)$, road holding $\left(\bar{\sigma}_{F_{z}}\right)$, and working space $\left(\bar{\sigma}_{x_{2}-x_{1}}\right)$, respectively. The stochastic objective functions are equivalent to the deterministic objective functions when $\alpha_{1}=$ $\alpha_{2}=\alpha_{3}=0$.

Tire stiffness is controlled primarily by the tire dimension and inflation pressure. Some analytical formulae can be found in the literature for its estimation [17-19]. In the following, the expression to estimate the vertical stiffness given in [17] is considered. The estimated results have been compared with more than 2000 measured values with a wide range of dimensions, and it turns out the expression has reasonable accuracy.

$$
k_{z}=0.00028 \cdot P \cdot \sqrt{(-0.004 A R+1.03) \cdot S_{N} \cdot\left(\frac{S_{N} \cdot A R}{50}+D_{R}\right)}+3.45
$$

where $P$ is the tire inflation pressure $(\mathrm{kPa}), S_{N}$ is the nominal section width $(\mathrm{mm}), D_{R}$ is the nominal rim diameter $(\mathrm{mm})$, and $A R$ is the aspect ratio.

The commonly used tire size nomenclature is described as follows. Take tire size 170/70R14 as an example, the 170 is the nominal section width $S_{N}$ in millimeters, 70 is the aspect ratio $A R, \mathrm{R}$ means radial construction, and 14 is the nominal rim diameter $D_{R}$ in inches.

Car manufacturers always provide different optional wheel sizes for the same vehicle. In order to keep a similar outer diameter, the tire size should always change together with the wheel. Moreover, the tire may be inflated to a pressure different from the nominal suggested value, and the tire inflating pressure also varies in presence of 
temperature variations and air leakages. Therefore, tire stiffness has to be considered affected by some uncertainty level.

Four tires, of two different sizes and two different constructions, suitable for the reference vehicle are listed in Table 2. In the table, the stiffness values computed by Equation (16) in the standard pressure range are reported.

Table 2. Vertical stiffness of standard tires and run-flat tires at different inflation pressure for the reference vehicle.

\begin{tabular}{lll}
\hline Tire size and type & $\begin{array}{l}k_{z} \text { at } 200 \mathrm{kPa} \\
(\mathrm{N} / \mathrm{m})\end{array}$ & $\begin{array}{l}k_{z} \text { at } 250 \mathrm{kPa} \\
(\mathrm{N} / \mathrm{m})\end{array}$ \\
\hline 175/70 R14 standard & 187894 & 226415 \\
215/45 R16 standard & 215521 & 260949 \\
175/70 R14 run-flat & 231109 & 278490 \\
215/45 R16 run-flat & 265091 & 320968 \\
\hline
\end{tabular}

The vertical stiffness of the run-flat tire (RFT) is higher in order to support the vehicle in the emergency condition of low/zero pressure. Based on the tests described in [20], the vertical stiffness of run-flat tire is about $23 \%$ higher than the standard tire.

Considering the values reported in Table 2, the tire vertical stiffness varies from $187894 \mathrm{~N} / \mathrm{m}$ to $320967 \mathrm{~N} / \mathrm{m}$. By assuming that the uncertainty of tire vertical stiffness follows a normal distribution, the mean value and the standard deviation of the tire stiffness distribution $\mathrm{read} \mu_{k_{1}}=254431 \mathrm{~N} / \mathrm{m}$ and $\sigma_{k_{1}}=22179 \mathrm{~N} / \mathrm{m}$. Substituting the value of $\sigma_{k_{1}}$ into Equation (14), the stochastic objective functions can be derived by Equation (15).

\section{Pareto-optimal solutions}

The Pareto-optimal sets in the design variables domain and objective functions domain are calculated for both the deterministic and stochastic formulations. Considering the fact that the Pareto-optimal set of the two objective functions is on the border of the projection of the surface that represents the Pareto-optimal set of the three objective functions problem [1], the optimization is performed using the $\varepsilon$-constraints method by considering two objective functions each time, namely, discomfort-road holding $\left(\sigma_{\ddot{x}_{2}}-\sigma_{F_{z}}\right)$, discomfort-working space $\left(\sigma_{\ddot{x}_{2}}-\sigma_{x_{2}-x_{1}}\right)$, working space-road holding $\left(\sigma_{x_{2}-x_{1}}-\sigma_{F_{z}}\right)$.

\subsection{The $\varepsilon$-constraints method to find Pareto-optimal solutions}

The $\varepsilon$-constraints method is one of the most effective technique to calculate the Pareto-optimal set [21]. The multi-objective optimization problem is converted into a single-objective minimization problem where the other objective functions become constraints, as shown in Equation (17).

$$
\begin{aligned}
& \min _{\mathbf{x} \in S} F_{1}(\mathbf{x}) \\
& F_{2}(\mathbf{x}) \leq \epsilon_{2}, F_{3}(\mathbf{x}) \leq \epsilon_{3}, \ldots, F_{k}(\mathbf{x}) \leq \epsilon_{\mathrm{k}}
\end{aligned}
$$

where $\epsilon_{i}, i=2, \ldots, k$ are the constraints levels for the objective functions.

Since only two objective functions are considered each time, the minimum for one objective function is calculated by varying the constraint level for the remaining objective function. The Pareto-optimal sets are calculated and presented in the following subsections.

\subsection{Pareto-optimal sets in the design variables domain}

The Pareto-optimal sets in the design variables domain are reported in Figure 2. The same robustness index $\left(\alpha_{1}=\alpha_{2}=\alpha_{3}\right)$ is set for all the stochastic objective functions for the sake of simplicity. In order to emphasize the difference between the robust optimization and deterministic optimization, the Pareto-optimal sets are obtained by considering different $\alpha$ values. The results of deterministic optimization are represented by the solid lines, which can be obtained by setting $\alpha_{1}=\alpha_{2}=\alpha_{3}=0$. The deterministic optimization only considers the mean value of the tire stiffness, which corresponds to the lowest robustness level. While the robust optimization also takes the uncertainty of tire stiffness into account by setting $\alpha_{1}=\alpha_{2}=\alpha_{3}=3$, which corresponds to a level of risk of about $0.1 \%$.

The points highlighted by markers $(\circ, \mathcal{\psi},+)$ on the Pareto-optimal sets for the deterministic optimization $(\alpha=$ $0)$ and points highlighted by markers $(\square, \diamond, \Delta)$ on the Pareto-optimal sets for the robust optimization $(\alpha=3)$ are also highlighted in Figure 3a) and b). The curves (1) and (3) of Figure 2 that go from the origin $\left(r_{2}=0, k_{2}=0\right)$ to the square marker $(\square)$ and circle marker $(\circ)$ represent the Pareto-optimal sets for the discomfort and road holding 
problem for robust and deterministic formulation respectively. The curves (2) and (4) that go from the square marker and circle marker to the upper bound of $r_{2}(5000 \mathrm{Ns} / \mathrm{m})$ represent the Pareto-optimal sets for the road holding and working space problem for robust and deterministic formulation respectively. The line (5) on the $r_{2}$ axis represent the Pareto-optimal sets for the discomfort and road holding problem for both robust and deterministic optimization. It is shown that the set of optimal solutions for robust optimization includes the optimal solutions for the deterministic optimization.

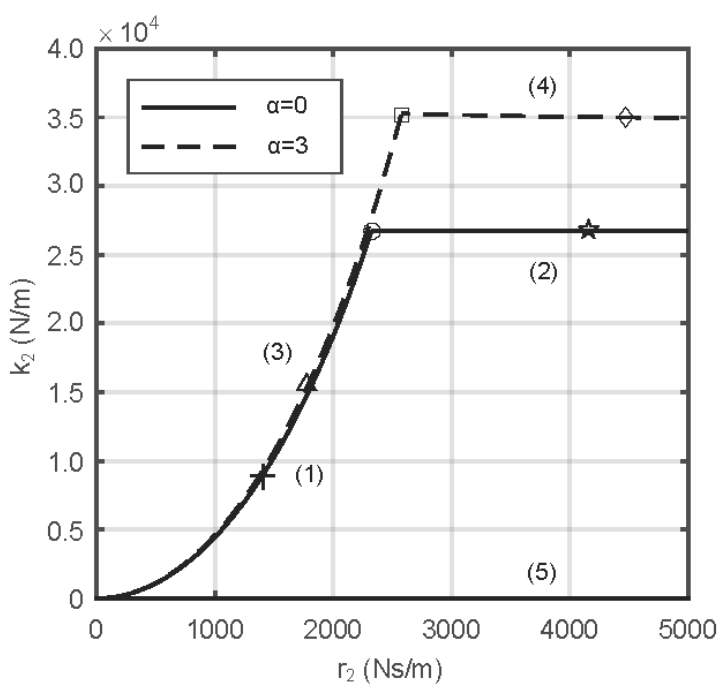

Figure 2. Pareto-optimal sets in the design variables domain.

\subsection{Pareto-optimal sets in the objective functions domain}

The Pareto-optimal sets for the objective functions combination $\sigma_{\ddot{x}_{2}}-\sigma_{F_{z}}$ and $\sigma_{x_{2}-x_{1}}-\sigma_{F_{z}}$ in the objective functions domain can be calculated by substituting the optimal design variables into the expressions of the deterministic objective functions in Equations (8)(9)(10). The results are reported in Figure 3.
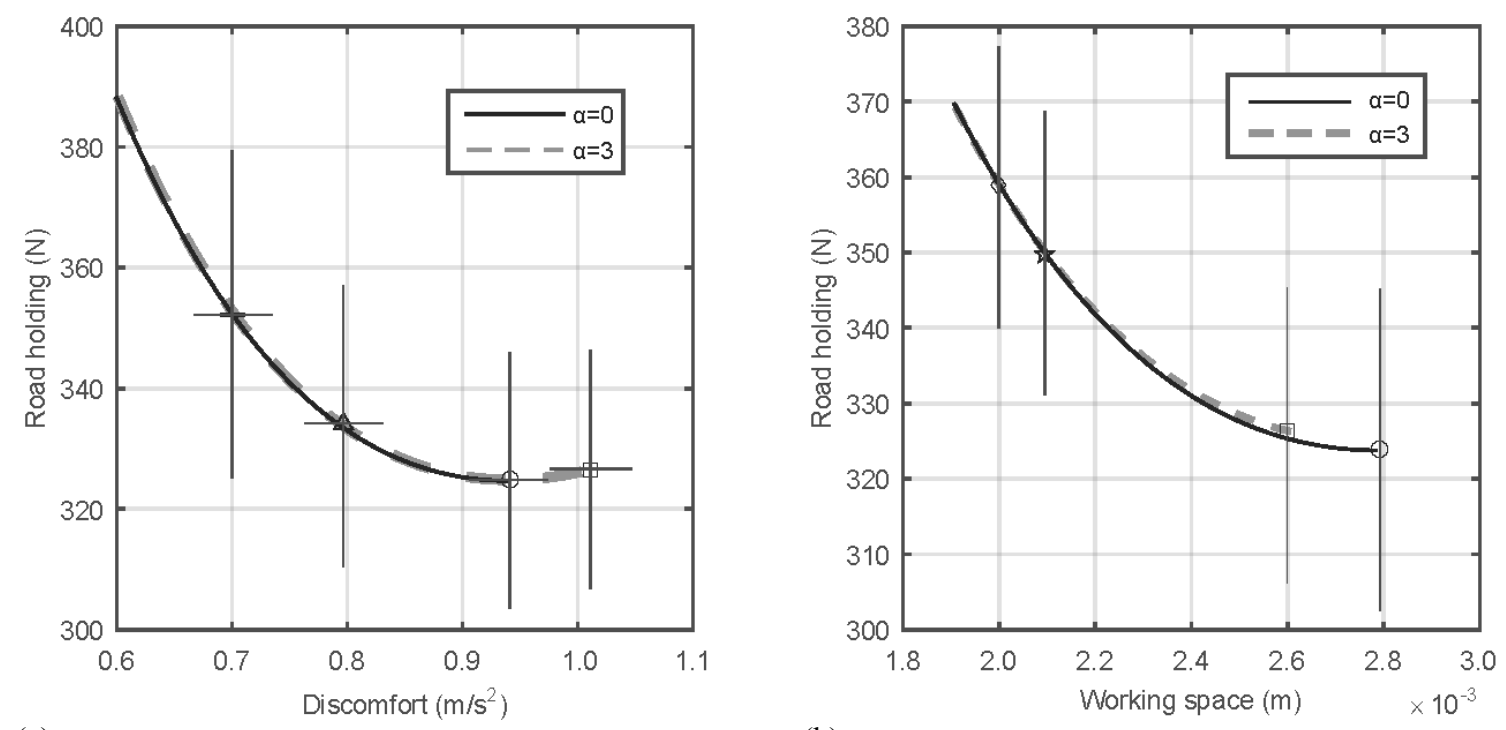

(b)

Figure 3. Pareto-optimal sets in the objective functions domain.

As shown in Figure 3, the optimal solutions obtained from the robust optimization $(\alpha=3)$ are very similar to the ones from the deterministic optimization $(\alpha=0)$. The standard deviations of the objective functions due to the uncertainty on tire stiffness are highlighted by the vertical and horizontal bars at each marked point. Table 3 provides a detailed comparison of the mean and standard deviation of discomfort $\left(F_{1}\right)$, road holding $\left(F_{2}\right)$, working space $\left(F_{3}\right)$ between the reference vehicle suspension (data in Table 1) and the solutions of the deterministic and robust optimization $(\alpha=0,3)$. It can be seen that both the two optimized suspensions have better performance on 
road holding and working space than the reference vehicle, and optimal results by robust optimization are less sensitive to the uncertainty of the tire stiffness.

Table 3. Comparison of mean and standard deviation of discomfort $\left(F_{1}\right)$, road holding $\left(F_{2}\right)$, working space $\left(F_{3}\right)$ between the reference vehicle suspension and the minimum of road holding for the considered optimizations.

\begin{tabular}{lllllll}
\hline & $\begin{array}{l}F_{1} \\
\left(\mathrm{~m} / \mathrm{s}^{2}\right)\end{array}$ & $\begin{array}{l}F_{2} \\
(\mathrm{~N} / \mathrm{m})\end{array}$ & $\begin{array}{l}F_{3} \\
(\mathrm{~mm})\end{array}$ & $\begin{array}{l}\sigma_{F_{1}} \\
\left(\mathrm{~m} / \mathrm{s}^{2}\right)\end{array}$ & $\begin{array}{l}\sigma_{F_{2}} \\
(\mathrm{~N} / \mathrm{m})\end{array}$ & $\begin{array}{l}\sigma_{F_{3}} \\
(\mathrm{~mm})\end{array}$ \\
\hline reference suspension & 0.74 & 374.5 & 4.2 & 0.02 & 32.7 & 0 \\
solution $\circ(\alpha=0)$ & 0.94 & 324.1 & 2.8 & 0.04 & 21.2 & 0 \\
solution $\square(\alpha=3)$ & 1.01 & 326.0 & 2.6 & 0.04 & 19.9 & 0 \\
\hline
\end{tabular}

\section{Conclusions}

In the paper, multi-objective optimization of passive suspension systems has been dealt with.

A two degree of freedom linear quarter-car model excited by the random irregular road has been used to model the vehicle dynamic behavior in the vertical direction. The considered suspension performance indices are road holding, discomfort and working space that have to be minimized, the design variables considered are the suspension equivalent stiffness and damping.

In order to optimize the dynamic performance (discomfort, road holding, and working space), the multiobjective deterministic optimization approach is utilized to find the Pareto-optimal solutions in terms of the design variables (suspension spring stiffness and damping coefficient).

However, the tire vertical stiffness is not constant, due to the use of run-flat tires, different tire sizes, and to the variation of tire pressure.

To account for the uncertainty in tire vertical stiffness, a multi objective robust optimization approach has been adopted to solve the problem. The Pareto-optimal solutions have been compared with the solutions obtained by considering the deterministic formulation. The results show that the robust optimization method can reduce the sensitivity of the optimal solutions to the variation of the tire stiffness, without significantly compromising the expected performance.

\section{References}

[1] Gobbi M, Mastinu G. Analytical description and optimization of the dynamic behaviour of passively suspended road vehicles. Journal of Sound and Vibration. 2001; 245(3):457-481.

[2] Gobbi M, Mastinu G. Symbolic multi-objective optimisation of the dynamic behaviour of actively suspended road vehicles. International Journal of Vehicle Design. 2002; 28(1-3):189-213.

[3] Ramakrishnan K, Yang L, Ballo F, et al. Multi-objective optimization of road vehicle passive suspensions with inerter. In: ASME Proceedings-18th International Conference on Advanced Vehicle Technologies. 2016. p.V003T01A007.

[4] Georgiou G, Verros G, Natsiavas S. Multi-objective optimization of quarter-car models with a passive or semi-active suspension system. Vehicle System Dynamics. 2007; 45(1):77-92.

[5] Stoll H, Reimpell J. Chassis technology: shock and vibration damper. Vogel Buchverlag Würzburg. 1989. (in German)

[6] Reimpell J, Betzler JW. Chassis technology: fundamentals. Vogel Buchverlag Würzburg. 2000. (in German)

[7] Gobbi M, et al. Optimal and robust design of a road vehicle suspension system. In: Proceedings of the 16th IAVSD Symposium. 2000; 33(4):3-22.

[8] Gobbi M, Levi F, Mastinu G. Multi-objective stochastic optimisation of the suspension system of road vehicles. Journal of Sound and Vibration. 2006; 298(4):1055-1072.

[9] Loyer B, Jezequel L. Robust design of a passive linear quarter car suspension system using a multi-objective evolutionary algorithm and analytical robustness indexes. Vehicle System Dynamics. 2009; 47(10):12531270.

[10] Jamali A, Salehpour M, Nariman-Zadeh N. Robust Pareto active suspension design for vehicle vibration model with probabilistic uncertain parameters. Multibody System Dynamics. 2013; 30(3):265-285.

[11] Kuerten D. Run-flat tires. In: IEEE Proceedings-Reliability and Maintainability Symposium; 1988. p.62-67.

[12] Sharp RS, Crolla DA. Road vehicle suspension system design-a review. Vehicle System Dynamics. 1987; 16(3):167-192.

[13] Dodds CJ, Robson JD. The description of road surface roughness. Journal of Sound and Vibration. 1973; 31(2):175-183.

[14] Mitschke M, Wallentowitz H. Vehicle dynamics. Springer-Verlag Berlin Heidelberg. 2004. (in German) 
[15] Müller PC, Schiehlen W. Linear vibrations: a theoretical treatment of multi-degree-of-freedom vibrating systems. Springer Science \& Business Media. 2012.

[16] Doebelin EO. Measurement systems: application and design (Chapter 2). McGraw-Hill; 1990.

[17] Gent AN, Walter JD. Pneumatic tire. 2005.

[18] Ballo F, Previati G, Gobbi M, Mastinu G. Tire-rim interaction, a semi-analytical tire model. Journal of Mechanical Design. 2018; 140(4):041401.

[19] Ballo F, Gobbi M, Mastinu G, Previati G. Motorcycle tire modeling for the study of tire-rim interaction. Journal of Mechanical Design. 2016; 138(5):051404.

[20] Heim R, Krause I, Weingaertner S. Runflat-technology and its impact on design and durability of wheels. SAE Technical Paper. 2007-01-1532.

[21] Mastinu G, Gobbi M, Miano C. Optimal design of complex mechanical systems: with applications to vehicle engineering. Springer Science \& Business Media; 2007.

(C) 2019 by the author(s). This work is licensed under a Creative Commons Attribution 4.0 International License (http://creativecommons.org/licenses/by/4.0/). Authors retain copyright of their work, with first publication rights granted to Tech Reviews Ltd. 